Recepción: 18 / 03 / 2018

Aceptación: 27 / 05 / 2018

Publicación: 05 / 06 / 2018

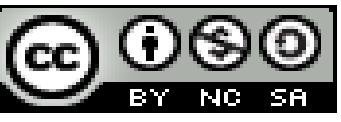

Ciencias de la salud Artículo de Revisión

\title{
Tratamiento y características del dolor postoperatorio en pacientes obstétricas que asisten al Centro de Salud Tosagua Tipo C
}
Treatment and characteristics of postoperative pain in patients obsterica to attend the center of health Tosagua Type $C$

\section{Tratamento e características da dor pós-operatória em pacientes obstétricas atendidas no Centro de Saúde Tipo Tosagua C}

\author{
Gaitán A. Barcia-Guerrero ${ }^{\mathrm{I}}$ \\ gaitan_73@hotmail.es \\ María S. Faubla-Zambrano II \\ silfaubla@gmail.com \\ María J. Cuenca-Jiménez III \\ majo_cuenca@hotmail.com
}

Correspondencia: gaitan_73@hotmail.es

\begin{abstract}
${ }^{\text {I } M a g i s t e r ~ e n ~ G e r e n c i a ~ E d u c a t i v a, ~ E s p e c i a l i s t a ~ e n ~ G e r e n c i a ~ y ~ P l a n i f i c a c i o ́ n ~ E s t r a t e ́ g i c a ~ e n ~ S a l u d, ~ D i p l o m a ~}$ Superior en Desarrollo Local y Salud, Magister en Gerencia de Salud para el Desarrollo Local, Doctor en Medicina y Cirugía, Docente e la Universidad Laica Eloy Alfaro de Manabí, Manta, Ecuador.

${ }^{\text {II } M e ́ d i c o ~ C i r u j a n o, ~ D o c e n t e ~ e ~ l a ~ U n i v e r s i d a d ~ L a i c a ~ E l o y ~ A l f a r o ~ d e ~ M a n a b i ́, ~ M a n t a, ~ E c u a d o r . ~}$

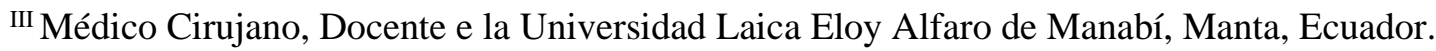




\section{Resumen}

En la paciente obstétrica, la percepción del dolor es el resultado de una experiencia única y multifactorial, la cual es influenciada por la ansiedad, las experiencias previas, los aspectos étnico-culturales y el medio ambiente. En este artículo, se evaluaron el tratamiento y las características del dolor postoperatorio en pacientes obstétricas que asisten al centro de salud Tosagua tipo C. La metodología fue de tipo descriptivo y de campo. La población estuvo constituida por 60 pacientes con puerperio quirúrgico, hospitalizadas en el servicio de obstetricia del Centro de Salud Tosagua Tipo C, ubicado en el Cantón de Tosagua de la Provincia de Manabí, las cuales fueron atendidas durante el segundo trimestre del año 2017. Los instrumentos utilizados fue una hoja de registro de datos personales de tipo sociodemográficos y la medición del dolor a través del instrumento tipo cuestionario que presenta Mc Caffery y Pasero (1999). Los datos aportados se procesaron estadísticamente considerando el análisis descriptivo y de medias a través del paquete estadístico del SPSS versión 23. Como resultado, se destaca que el dolor moderado presento el mayor porcentaje con el 53\% del total de las pacientes investigadas. Entre sus conclusiones se pudo determinar que la intensidad del dolor máximo evaluado durante las primeras 24 horas, en una escala del 1 al 10, fue de 5.9 y la menor manifestación de dolor se presentó con una media de 4.8 de intensidad.

Palabras clave: tratamiento del dolor; características del dolor; postoperatorio y parto.

\section{Abstract}

A In the obstetric patient, the perception of pain is the result of a unique experience and multifactorial, which is influenced by anxiety, previous experiences, the ethno-cultural aspects and the environment. In this article, were evaluated treatment and characteristics of postoperative pain in obstetric patients attending health Tosagua type C. The methodology was descriptive and field. The population consisted of 60 patients with surgical puerperium, hospitalized in the service of obstetrics from the center of health Tosagua type $\mathrm{C}$, located in the Canton of Tosagua province of Manabí, which were attended during the second quarter the year 2017. The instruments used was a sheet of personal type socio-demographic data and the measurement of pain through the instrument type questionnaire presenting Mc Caffery and Pasero (1999). The data were processed statistically whereas the descriptive and analysis mean using the SPSS 
statistical package version 23. As a result, stands that moderate pain presented the highest percentage with $53 \%$ of the total of the investigated patients. Among its conclusions, it was determined that the maximum pain evaluated during the first 24 hours, on a scale of 1 to 10 , was 5.9 and the lower manifestation of pain was presented with an average of 4.8 of intensity.

Key words: pain, characteristics of pain treatment, postoperative and postpartum.

\section{Resumo}

Em pacientes obstétricas, a percepção da dor é o resultado de uma experiência única e multifatorial, que é influenciada pela ansiedade, experiências anteriores, aspectos étnico-cultural e do meio ambiente. Neste artigo, o tratamento e as características da dor pós-operatória em pacientes obstétricas que frequentam o centro de saúde tipo Tosagua foram avaliados C. A metodologia foi descritiva e de campo. A população foi composta por 60 pacientes com puerpério cirúrgico internado no Departamento de Obstetrícia Centro de Saúde Tosagua tipo C, localizada em Canton Tosagua da província de Manabí, que foram abordados durante o segundo trimestre de 2017. O instrumento utilizado era uma folha de registo de dados pessoais de tipo sociodemográfico e medição de dor através do instrumento tipo questionário tendo Mc Caffery e Pasero (1999). Os dados foram processados estatisticamente fornecida considerando a análise descritiva do meio utilizando o pacote estatístico SPSS versão 23. Como resultado, salienta que a dor moderada teve a maior percentagem de $53 \%$ de todos os pacientes investigados. Entre as suas conclusões determinou-se que a intensidade da dor máxima avaliada durante as primeiras 24 horas, numa escala de 1 a 10, foi de 5,9 e a menor manifestação da dor foi apresentada com uma média de 4,8 intensidade.

Palavras chave: tratamento da dor; características da dor; pós-operatório e parto.

\section{Introducción}

Para el cuerpo físico, apunta la Asociación Internacional para el estudio del dolor (2010), que el dolor es un mecanismo de expresión fisiológica de desagravio o defensa, frente a una agresión real o con potencial similar, pero con la expresión y la reacción del individuo hacia ella, ocurre con la interpretación emocional y social estructurada por la cultura. La misma asociación, explica que, para el cuerpo físico, el dolor es un mecanismo de expresión fisiológica de desagravio o 
defensa, frente a una agresión real o con potencial similar, pero con la expresión y la reacción del individuo hacia ella, ocurre con la interpretación emocional y social estructurada por la cultura.

Al respecto, Covarrubias et al (2006), afirma que, en la paciente obstétrica, la percepción del dolor es el resultado de una experiencia única y multifactorial, la cual es influenciada por la ansiedad, las experiencias previas, los aspectos étnico-culturales y el medio ambiente. En tanto, que el dolor del trabajo de parto involucra una complejidad de respuestas neurocomportamentales al estímulo álgico y suministra una característica personal y única al dolor que se siente. La relación de causa y efecto en esa situación no siempre corresponde, en la práctica, a una respuesta clínica, porque lo que importa en ese caso, es entender el dolor vivido por la parturiente y amenizárselo. En ese sentido, Mazoni y Carvalho (2008), argumentan en que muchas veces el dolor se manifiesta incluso en la ausencia de las agresiones tisulares vigentes.

Al respecto, cabe destacar que el dolor durante el trabajo de parto es ocasionado por las contracciones de los músculos y la presión sobre el cuello uterino. Este dolor se puede sentir como un cólico intenso en el abdomen, las ingles y la espalda, y también como una sensación de malestar general. Algunas mujeres también experimentan dolor en los costados o en los muslos. Otras causas del dolor durante el trabajo de parto incluyen la presión que ejerce la cabeza del bebé sobre la vejiga y los intestinos, y el estiramiento del canal de parto y la vagina. Además, el dolor durante el trabajo de parto es diferente para cada mujer. El dolor varía mucho de una mujer a otra, e incluso de un embarazo a otro. Las mujeres sienten el dolor del trabajo de parto de distintas formas. Para algunas, se parece al dolor menstrual; para otras, es una presión intensa; y, para otras, son oleadas muy fuertes que se asemejan a los cólicos abdominales. A menudo, no es el dolor en cada contracción lo que las mujeres consideran más duro, sino el hecho de que las contracciones son constantes y, conforme el trabajo de parto avanza, hay cada vez menos tiempo entre una contracción y otra para relajarse.

Asimismo, Maldonado (2002), expresa que el parto, a diferencia del embarazo que por un largo período favorece la adaptación gradual de los cambios, se caracteriza como siendo un evento que provoca cambios abruptos e intensos, que marcan algunos niveles de simbolización, como la intensidad del dolor y la imprevisibilidad, causando el sufrimiento, la ansiedad y la inseguridad. En el caso específico del trabajo de parto, el dolor produce cambios fisiológicos maternos que 
pueden afectar el bienestar materno y fetal, en la que su manejo inadecuado postoperatorio en postparto o postcesárea puede afectar de forma significativa el bienestar de la madre y el neonato; esto es debido a que en presencia de dolor se retrasa la deambulación, el tiempo de inicio de la ingesta y la ventilación presenta un patrón restrictivo que condiciona a la acumulación de secreciones.

Estas alteraciones, en conjunto, favorecen el desarrollo de complicaciones como lo son el íleo, atelectasias, neumonía, tromboembolismos; así mismo, la liberación de catecolaminas inhibe la secreción de oxitocina, con lo cual la producción de leche materna disminuye o se suspende. En base a este planteamiento, es de señalar que el presente artículo detalla el En este artículo, se evaluaron el tratamiento y las características del dolor postoperatorio en 60 pacientes obstétricas que asisten al centro de salud Tosagua tipo $\mathrm{C}$.

\section{Metodología}

La metodología fue de tipo descriptivo y de campo, que de acuerdo a Hernández y et al (2010), refiere a la descripción del fenómeno de estudio tal como se presenta y de campo en tanto los datos se obtuvieron en el mismo sitio de la investigación. La población estuvo constituida por 60 pacientes con puerperio quirúrgico, hospitalizadas en el servicio de obstetricia del Centro de Salud Tosagua Tipo C, ubicado en el Cantón de Tosagua de la Provincia de Manabí, las cuales fueron atendidas durante el segundo trimestre del año 2017. Los instrumentos utilizados fue una hoja de registro de datos personales de tipo sociodemográficos y la medición del dolor a través del instrumento tipo cuestionario que presenta Mc Caffery y Pasero (1999), el cual mide la intensidad del dolor, así como, el nivel de satisfacción con el manejo del dolor por parte de los médicos y el personal de enfermería. Es un instrumento confiable y está compuesto por 9 reactivos los cuales se miden de diferente manera, los primeros cuatro miden la presencia e intensidad del dolor por medio de la escala visual numérica con valores de 0 a 10 , donde 0 es ausencia de dolor y 10 es el máximo dolor. Los datos aportados se procesaron estadísticamente considerando el análisis descriptivo y de medias a través del paquete estadístico del SPSS versión 23. 


\section{Análisis y discusión de los resultados}

A continuación, se presenta los resultados más representativos de la información obtenida a partir de la hoja de registro que solicitaba información a las pacientes acerca de los datos sociodemográficos y obstétricos y de la aplicación del instrumento tipo cuestionario que presenta Mc Caffery y Pasero (1999).

\section{Características de las mujeres participantes}

En la tabla y grafico 1, se presentan las edades y el nivel de escolaridad de la población estudiada, donde se observa que las edades fluctuaron entre 15 y 42 años, con una media entre 17-22 años con una desviación estándar de 2,4, de los cuales 27 que represento el $45 \%$ del total fueron primigestas y 33 fueron multíparas representadas en el 55\% y su nivel de escolaridad de 9 años con una desviación estándar de 2,2.

\section{Tabla 1}

\section{Población estudiada}

\begin{tabular}{l|c}
\multicolumn{1}{c|}{$\begin{array}{c}\text { Análisis/ } \\
\text { Alternativas }\end{array}$} & Media \pm DE \\
\hline Edad & $(\mathbf{1 7 - 2 2 )} \pm \mathbf{2 , 4}$ \\
Nivel de Escolaridad & $\mathbf{9 \pm 2 , 2}$ \\
& \\
\hline
\end{tabular}

Fuente: Elaboración propia. 


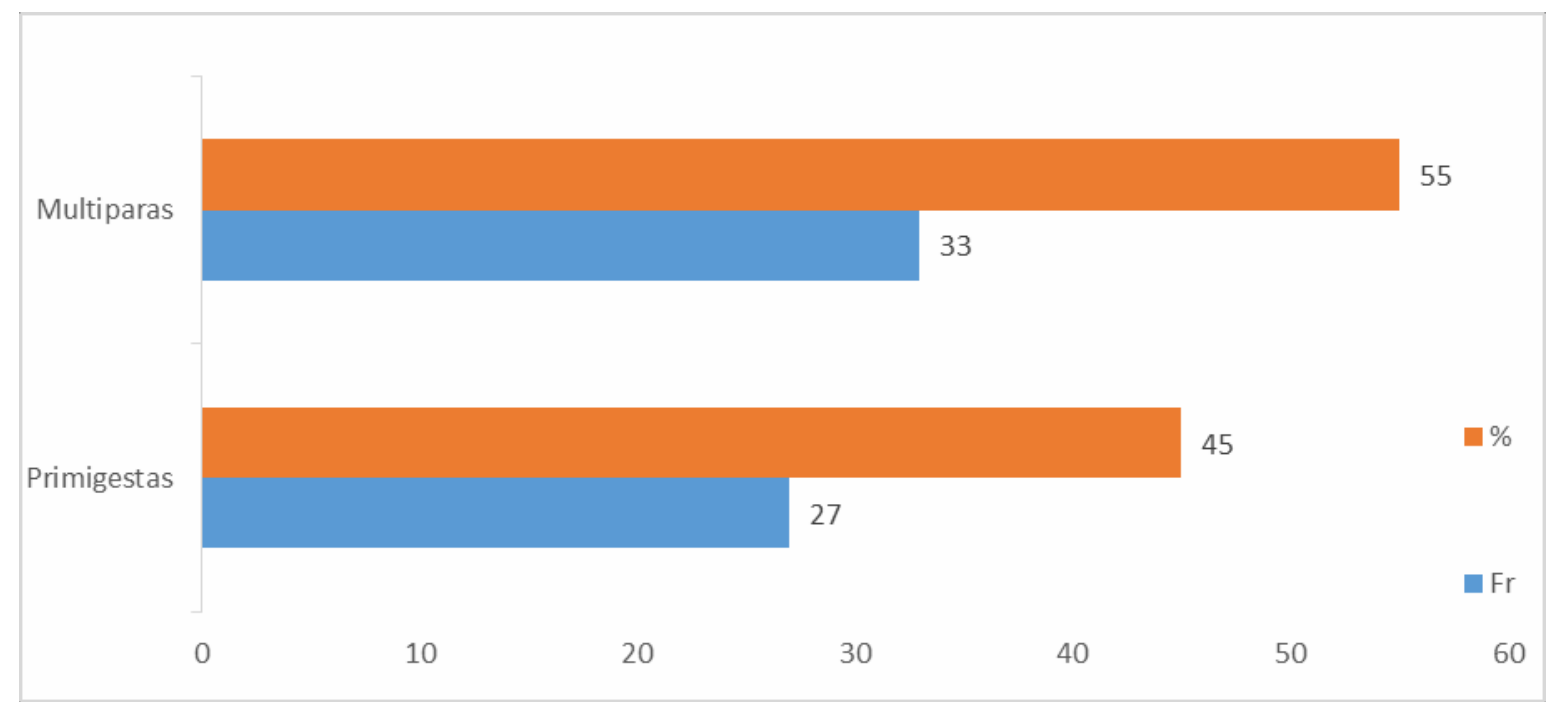

\section{Grafico 1. Población estudiada}

En la tabla y grafico 2, se presentan los resultados del análisis de los datos que dieron a conocer acerca si habían recibido las pacientes obstétricas orientación preoperatoria en un $78.6 \%$ de las cuales el $45.6 \%$ indico que recibió la orientación del médico, anestesiólogo y enfermera y un 4.7 señalo que la recibieron del médico y del anestesiólogo.

\section{Tabla 2}

\section{Análisis Descriptivo del Indicador Orientación preoperatoria}

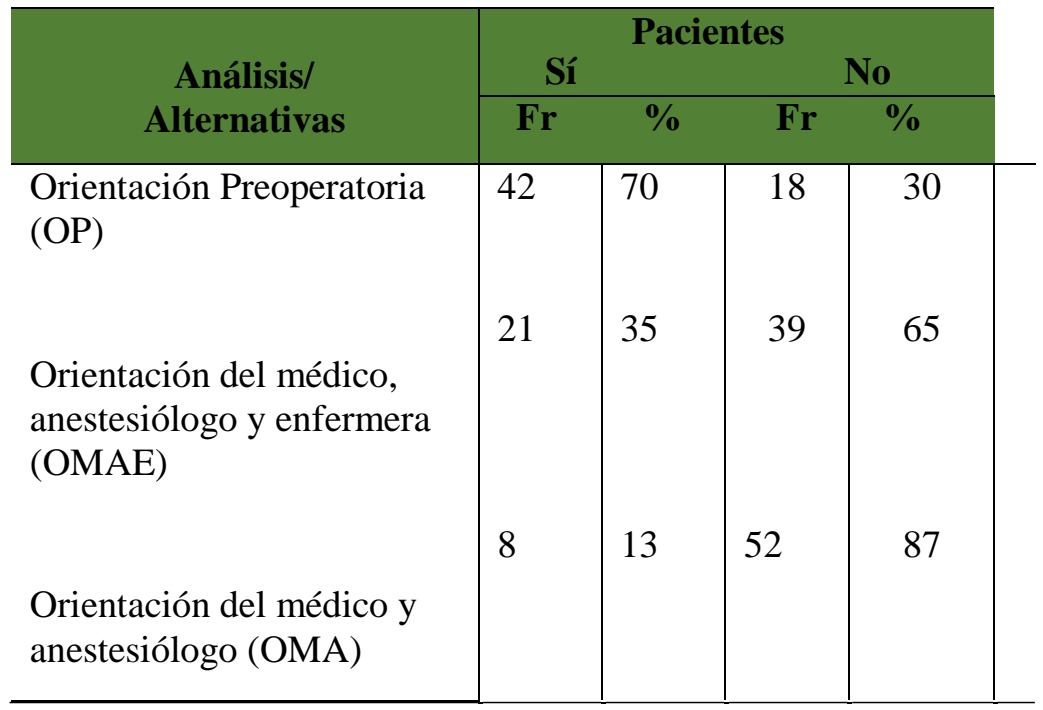




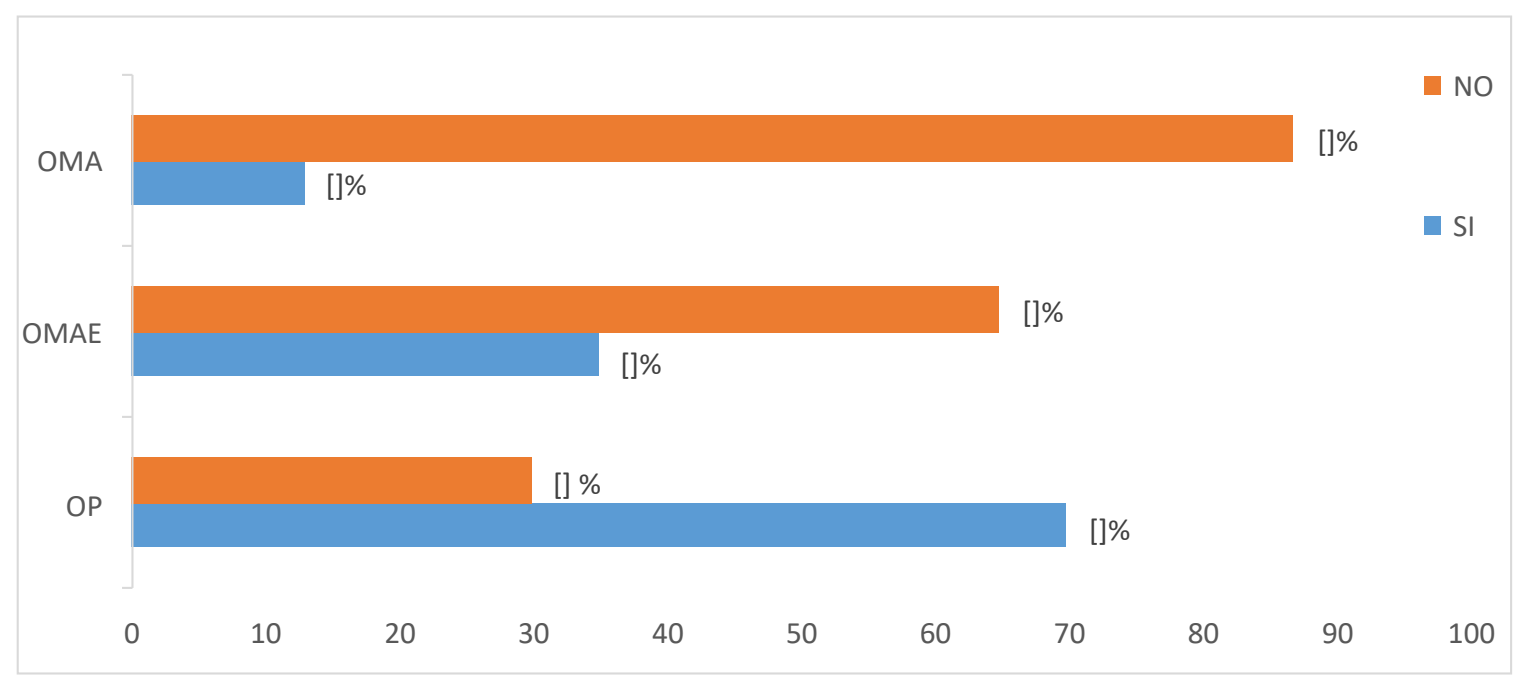

Grafico 2. Análisis Descriptivo del Indicador Orientación preoperatoria

\section{Características del dolor según el test de Aplicaa Mc Caffery y Pasero (1999)}

En la tabla y grafico 3, se presenta el análisis de los datos que permitieron evaluar el dolor máximo en las primeras 24 horas que refirieron las pacientes hospitalizadas en el servicio de obstetricia del Centro de Salud Tosagua tipo C, en la que se indica una media de 5.9 con una desviación estándar de 0.9 de dolor máximo durante las primeras 24 horas. La manifestación de dolor en las primeras 24 horas presento una media de 4.8 con una desviación estándar de 1,7, ambos en una escala del 1 al 10. 


\section{Tabla 3}

\section{Análisis Descriptivo del Indicador Dolor}

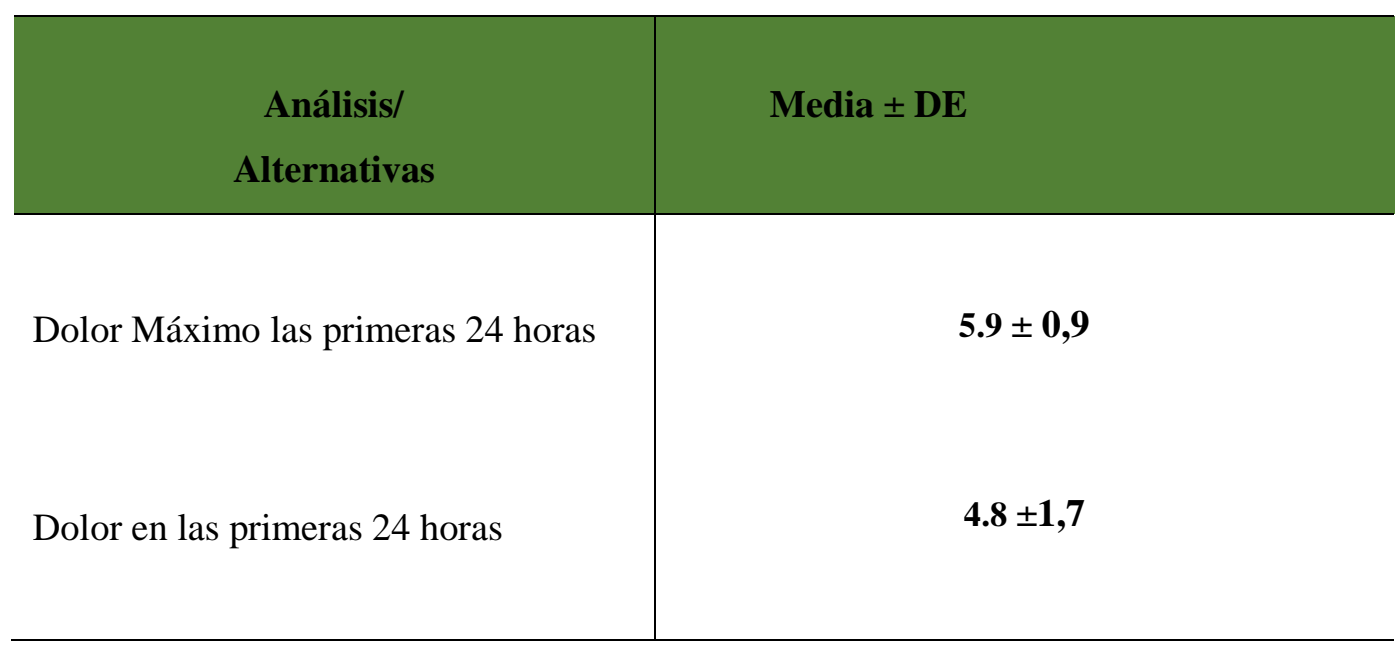

\section{Fuente: Elaboración propia.}

En la tabla y grafico 4, se presentan los resultados del análisis de los datos que permitieron evaluar la intensidad del dolor en las pacientes hospitalizadas en el servicio de obstetricia del Centro de Salud Tosagua tipo C, en la misma se indica que el dolor moderado presento el mayor porcentaje con el 53\% del total de las pacientes investigadas. Seguido del 33\% que manifestaron haber tenido dolor severo. Los menores porcentajes se indicaron para pacientes que manifestaron ausencia del dolor y dolor leve con el $3 \%$ y $9 \%$ respectivamente.

Tabla 4

Análisis Descriptivo del Indicador Intensidad del dolor

\begin{tabular}{|c|c|c|c|c|}
\hline \multirow{3}{*}{$\begin{array}{c}\text { Análisis/ } \\
\text { Alternativas }\end{array}$} & \multicolumn{4}{|c|}{ Pacientes } \\
\hline & \multicolumn{2}{|c|}{ Sí } & \multicolumn{2}{|c|}{ No } \\
\hline & $\mathbf{F r}$ & $\%$ & $\mathbf{F r}$ & $\%$ \\
\hline Ausencia del dolor & 2 & 3 & 58 & 97 \\
\hline Dolor Leve & 6 & 9 & 54 & 91 \\
\hline Dolor Moderado & 32 & 53 & 28 & 47 \\
\hline Dolor Severo & 20 & 33 & 40 & 67 \\
\hline
\end{tabular}

Fuente: Elaboración propia. 


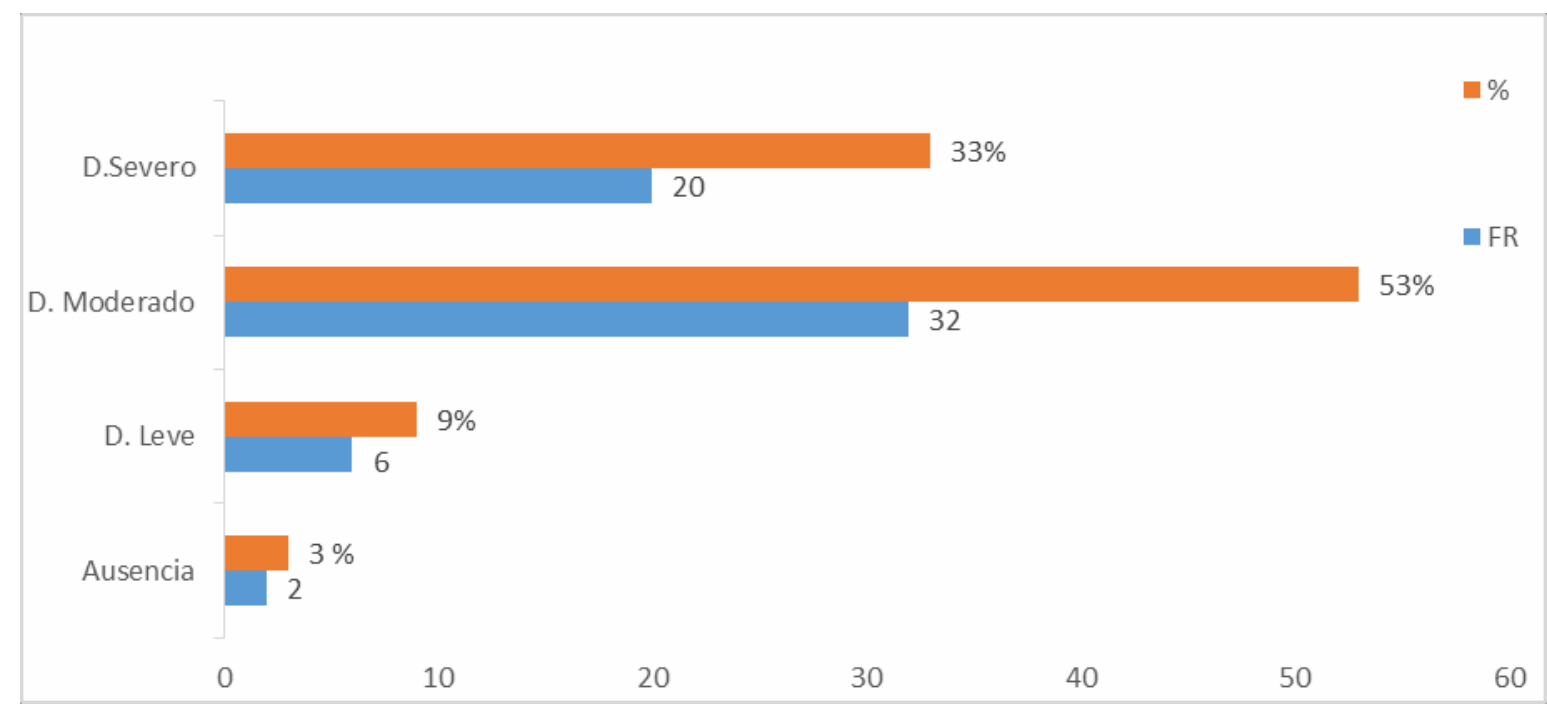

\section{Grafico 4. Análisis Descriptivo del Indicador Intensidad del dolor}

En la tabla y grafico 5, se presentan los resultados del análisis de los datos que permitieron evaluar el indicador tiempo de espera para recibir analgésico en las pacientes hospitalizadas en el servicio de obstetricia del Centro de Salud Tosagua tipo C, en la que se indica que en el tiempo de espera para recibir el analgésico cuando reportaron las mujeres tener dolor, tardó menos de 15 minutos en el $66 \%$ del total. Seguido del $18 \%$ que indicaron haber recibido atención después de 15 minutos. El menor porcentaje con el $18 \%$ señalo la alternativa no solicitaron medicamento ni tratamiento para el dolor.

\section{Tabla 5}

\section{Análisis Descriptivo del Indicador Tiempo de espera}

\begin{tabular}{|c|c|c|c|c|}
\hline & \multicolumn{4}{|c|}{ Pacientes } \\
\hline Alternativas & $\mathbf{F r}$ & $\%$ & Fra & $\% \mathbf{a}$ \\
\hline Mayor a 15 minutos & 12 & 18 & 12 & 18 \\
\hline Menos de 15 minutos & 40 & 66 & 52 & 84 \\
\hline $\begin{array}{l}\text { No solicitaron } \\
\text { medicamentos }\end{array}$ & 8 & 12 & 60 & 100 \\
\hline
\end{tabular}

Fuente: Elaboración propia. 


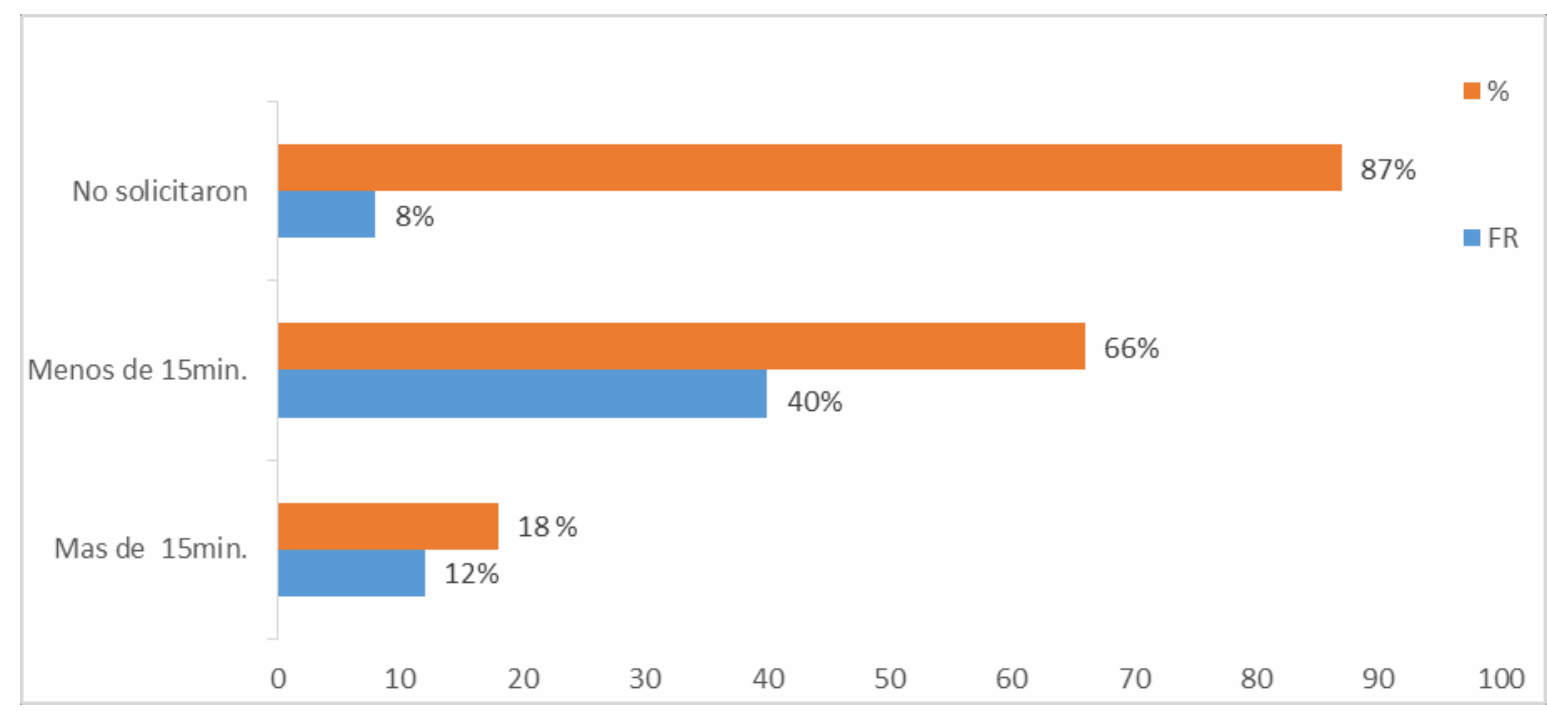

\section{Grafico 5. Análisis Descriptivo del Indicador Tiempo de espera}

En la tabla y grafico 6, se presentan los resultados del análisis de los datos que permitieron evaluar el indicador satisfacción con el manejo del dolor en las pacientes hospitalizadas en el servicio de obstetricia del Centro de Salud Tosagua tipo C, en la que se indica que la satisfacción con el manejo del dolor en las mujeres con cesárea iterativa obtuvo una media de 7.2 con una desviación estándar de 0.5, ligeramente mayor que las que tenían cesárea anterior y su primera cesárea. La satisfacción según la indicación médica fue mayor en las que tuvieron una cesárea electiva y según la técnica quirúrgica Beck y Kerr obtuvieron una media muy similar. 


\section{Tabla 6}

Análisis Descriptivo del indicador Satisfacción con el manejo del Dolor

\begin{tabular}{l|c}
\multicolumn{1}{c|}{$\begin{array}{c}\text { Análisis/ } \\
\text { Alternativas }\end{array}$} & Media \pm DE \\
\hline & \\
Cesárea iterativa & $\mathbf{7 . 2} \pm \mathbf{0 . 5}$ \\
Cesaría anterior & $\mathbf{5 . 4} \pm \mathbf{1 . 6}$ \\
Primera cesárea & $\mathbf{8 . 6 \pm 0 . 9}$ \\
& \\
\hline
\end{tabular}

Fuente: Elaboración propia.

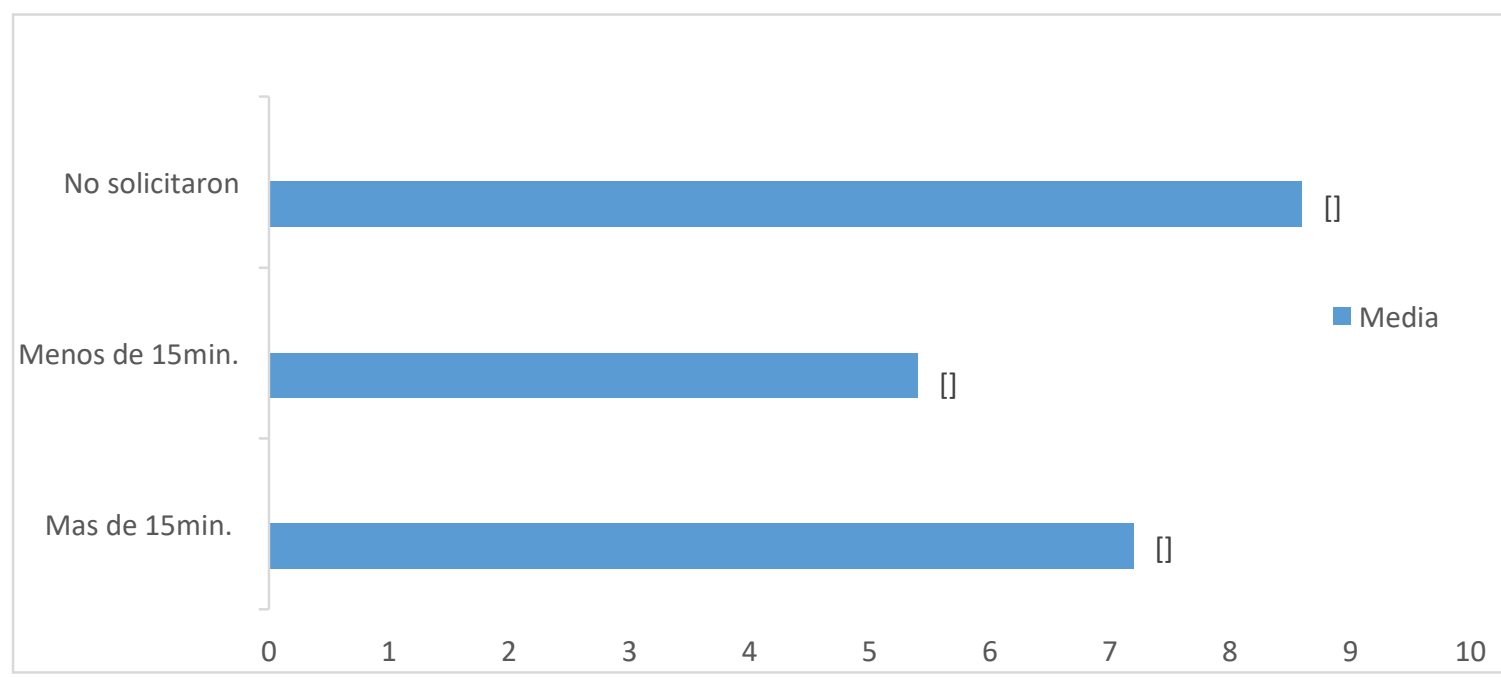

Grafico 6. Análisis Descriptivo del indicador Satisfacción con el manejo del Dolor

Asimismo, se pudo determinar que las mujeres que reportaron el dolor máximo en 24 horas fueron las que no recibieron orientación preoperatoria contra las que la recibieron (7.33 vs 6.05, p $=.01)$, Se constató que las mujeres que estaban más satisfechas con el manejo del dolor, fueron las que recibieron orientación preoperatoria respecto a las que no las recibieron $(\mathrm{p}=.0)$. 
Asimismo, las mujeres que se les realizó la cesárea de urgencia presentaron mayor intensidad del dolor las primeras 24 horas, el dolor promedio y la interferencia entre la actividad general.

\section{DESARROLLO}

La elección para tratar el dolor del parto, puede ser de forma natural o mediante el uso de medicamentos, pues generalmente suele hacerse una mezcla de ambos. Al respecto, se presentan el manejo y forma natural y por medicamento para tratar el dolor durante el parto.

\section{Forma Natural del trato del dolor durante el parto.}

Elegir una ruta natural, no recibirá ningún medicamento para el dolor. Igualmente puede recibir atención médica, pero va a depender de los métodos naturales de tratamiento del dolor en lugar de los medicamentos. Estos incluyen técnicas de respiración especiales y otros enfoques. Es posible que desee planificar excepciones para obtener medicamentos si hay problemas. Está bien si su plan inicial de un parto natural cambia y termina queriendo medicamento para el dolor.

Método Bradley. Este enfoque se centra en un embarazo saludable y activo y el descanso mental en el trabajo de parto. Esta práctica sólo cree en el parto natural y no se puede utilizar con medicamentos. Puede hacerse una excepción para problemas graves.

El parto en agua y el hipnoparto. Son otros enfoques naturales cada vez más populares. Algunas mujeres consideran que el agua es un entorno relajante y una entrada más natural para el bebé. Existe poca investigación sobre la seguridad y el valor de este enfoque. El hipnoparto se centra en un estado de ánimo en donde el parto es indoloro. Se le enseña a desprenderse del miedo al trabajo de parto y tener un parto tranquilo. Entre otras actividades naturales que puede intentar para el manejo del dolor incluyen: Cambiar de posición, salir a caminar, masaje, yoga o estiramiento, utilización de una almohadilla caliente o una toalla fría, tomar un baño o ducha, escuchar música, meditación o visualización y cualquier otra actividad que la ayude a relajarse o distraerse.

Alternativas no farmacológicas. Pueden ser utilizadas como complemento para retrasar la administración de la anestesia medicamentosa e incluyen técnicas como la hipnosis, reiki, yoga y 
esferoterapia, las que son realizadas en la sala de preparto, también son alternativas la terapia de calor y frío, el hipnotismo, la musicoterapia y aromaterapia. En un estudio realizado por Olapour et al (2013), para el alivio del dolor después de la cesárea, encontraron una reducción significativa en el dolor después de la inhalación de lavanda y podría ser utilizado como una parte del tratamiento analgésico multimodal después de una cesárea.

\section{Uso de medicamentos para el tratamiento del dolor durante el trabajo de parto}

El estándar de manejo es una analgesia de la columna vertebral, que para Whizar (2009), significa controlar el dolor desde la cintura hacia abajo. Los tipos de anestesia que pueden indicarse durante el trabajo de parto y el parto mismo son:

Anestesia peridural: es el tipo más frecuente de analgesia en el trabajo de parto es la denominada peridural en la que el médico instala un catéter por fuera del saco de líquido que rodea la médula espinal, denominado espacio epidural, mediante el que inyecta un medicamento que insensibiliza o bloquea la sensibilidad para que el paciente no experimente dolor. A través de este catéter, la paciente puede recibir más medicamentos en la medida en que lo necesite sin necesidad de una nueva punción. Es precisa su indicación terapéutica en ciertas complicaciones del embarazo como la hipertensión inducida por este, la presentación de nalgas y la acción uterina incoordinada. La punción para llegar al espacio peridural se efectúa entre los espacios vertebrales lumbares tercero y cuarto o entre el cuarto y el quinto, obteniéndose el bloqueo mediante variadas concentraciones de drogas analgésicas locales y narcóticos.

Anestesia combinada espinal-epidural: en ella se combina una anestesia espinal llamada también raquídea con una anestesia peridural para aprovechar las ventajas de ambas. Este tipo de anestesia comienza a hacer efecto inmediatamente y funciona bien para procedimientos más cortos mientras que la anestesia epidural es más indicada para procedimientos más prolongados. De allí que la combinación de ambas suele ser una buena opción para manejar el dolor. En esta técnica, ideada por el anestesiólogo argentino Torrieri (2010) se aúnan la rapidez de acción de la analgesia raquídea con la flexibilidad del bloqueo epidural. Es posible prolongar, si así lo requieren las circunstancias, un periodo indoloro en la etapa posterior al parto lo mismo que proporcionar inmediata analgesia a una operación cesárea no prevista. 


\section{Conclusiones}

Las conclusiones que se exponen se corresponden a un total de 60 pacientes que fueron hospitalizadas en el servicio de obstetricia del Centro de Salud Tosagua tipo C, en las que se pueden mencionar:

-Las edades de las pacientes investigadas fluctuaron entre 15 y 42 años, con una media entre 1722 años, en la que se constató que el 45\% del total fueron primigestas y el resto manifestaron ser multíparas.

-Se constató en un alto porcentaje que las pacientes de obstétrica recibieron orientación preoperatoria y en menor proporción manifestaron haber recibido orientación por parte del médico, anestesiólogo y enfermera.

-La intensidad del dolor máximo evaluado durante las primeras 24 horas, en una escala del 1 al 10, entre las pacientes investigadas fue de 5.9 y la menor manifestación de dolor se presentó con una media de 4.8 de intensidad.

-Durante la hospitalización, la mayor manifestación de dolor en las pacientes hospitalizadas fue moderado en el $53 \%$ y en un $33 \%$ de dolor severo.

- El tiempo de espera para recibir el analgésico cuando reportaron las mujeres tener dolor, tardó menos de 15 minutos según el 66\% de las opiniones de las investigadas, en la cual solo el $18 \%$ señalo la alternativa no solicitaron medicamento ni tratamiento para el dolor.

- La satisfacción con el manejo del dolor en las mujeres con cesárea iterativa fue ligeramente mayor que las que tenían cesárea anterior y su primera cesárea. La satisfacción según la indicación médica fue mayor en las que tuvieron una cesárea electiva y según la técnica quirúrgica Beck y Kerr obtuvieron una media muy similar.

- Se determinó una relación no significativa entre la satisfacción con el manejo del dolor por el personal de salud con la presencia de dolor durante la entrevista a las pacientes, indicando que no existe incidencia de asociación del dolor máximo en las 24 horas y el dolor promedio. 


\section{Referencias Bibliográficas}

Covarrubias-Gómez A, Silva-Jiménez A, Nuche-Cabrera E, Téllez-Isaía

IASP (2010). International Association for the Study of Pain. Disponível en: ttps://en.wikipedia.org/wiki/International_Association_for_the_Study_of_Pain. Consultado en diciembre del 2017

M (2006). El manejo del dolor postoperatorio en obstetricia: ¿Es seguro? Revista

Maldonado MTP - Aspectos Psicológicos da Gravidez do Parto e do Puerpério, em: Maldonado MTP - Psicologia da Gravidez, 16ª Ed. Petrópolis, Vozes, 2002; 48-60.

Mazoni SR, Carvalho EC - Dor de parto: considerações históricas e conceituais. Rev Dor, 2008;9:1176-1182.

McCaffery M y Pasero C. (1999). Pain: Clinical Manual. St. Louis: Mosby;

Mexicana de Anestesiología.

Olapour A, Behaeen K, Akhondzadeh R (2013). The effect of inhalation of aromatherapy blend containing lavander essential oil on cesarean postoperative pain. Anesth Pain. 203-207

Paladino M A (2003). Accidentes, incidentes y complicaciones intraoperatorias. Rev Arg Anest.

Torrieri, A. (2010). Tratamiento del dolor en el parto. Ahttps://www.google.com.ec/search?q=Alberto+Torrieri+y+el+parto\&oq=albert\&aqs=chrome.1. 69i5912j69i57j013.3338j0j7\&sourceid=chrome\&ie=UTF-8

Whizar Lugo V (2009). Prevención en Anestesiología. Anestesia en México. 